\title{
Kernos
}

Revue internationale et pluridisciplinaire de religion grecque antique

33 | 2020

Varia

\section{La Cité des regards. Autour de François Lissarrague}

\section{Anne-Françoise Jaccottet}

\section{OpenEdition \\ Journals}

\section{Édition électronique}

URL : https://journals.openedition.org/kernos/3628

DOI : 10.4000/kernos.3628

ISSN : 2034-7871

\section{Éditeur}

Centre international d'étude de la religion grecque antique

\section{Édition imprimée}

Date de publication : 31 décembre 2020

Pagination : 346-350

ISBN : 978-2-87562-264-8

ISSN : 0776-3824

\section{Référence électronique}

Anne-Françoise Jaccottet, « La Cité des regards. Autour de François Lissarrague », Kernos [En ligne], 33 | 2020, mis en ligne le 31 décembre 2020, consulté le 05 décembre 2022. URL : http:// journals.openedition.org/kernos/3628; DOI : https://doi.org/10.4000/kernos.3628

Ce document a été généré automatiquement le 5 décembre 2022

Tous droits réservés 


\title{
La Cité des regards. Autour de François Lissarrague
}

\author{
Anne-Françoise Jaccottet
}

\section{RÉFÉRENCE}

Vasiliki ZACHARI, Élise LEHOUX, Noémie HOSOI (dir.), La Cité des regards. Autour de François Lissarrague, Rennes, Presses Universitaires de Rennes, 2019. 1 vol. $17 \times 24,5$ cm, 306 p. + 106 fig. (Art et société). ISBN : 978-2-7535-7609-4.

Le jeu de mot allusif du titre le dit d'entrée : cet ouvrage est un bouquet d'hommages offert à François Lissarrague, cet inlassable chasseur d'images, de sens, de liens, ce scrutateur du regard, des regards, ceux que les images mettent en scène et nous renvoient, ceux que nous portons sur les images. Les dix-neuf textes réunis sont autant de regards personnels sur l'homme, sur sa recherche et son enseignement de la part de celles et ceux qui ont suivi ses séminaires et ont été " captivés par son approche, sa méthode, la fluidité de son discours et par ce que son regard nous invite à voir » (p. 9). Le lien entre les différents textes se tissera donc entre les lignes, par l'ancrage méthodologique plus que par une thématique réflexive, par des démarches analytiques qui, reprenant les axes favoris du maître, forment la trame et le fondement de ces offrandes. C'est ainsi que se croisent et s'entremêlent en deçà et au-delà des sujets particuliers, l'importance de l'objet porteur d'images, et sa manipulation, dans le cas des vases, induisant des changements de perspectives, le dialogue des différentes images sur le support et avec le support, l'attention portée au détail, à la place des figures, la réflexion sur les objets et leur sens dans la construction de l'image, l'analyse des absences: autant de marques que l'on retrouve dans la foisonnante bibliographie de François Lissarrague présentée en exergue (p. 13-26), de pistes qui relient entre eux

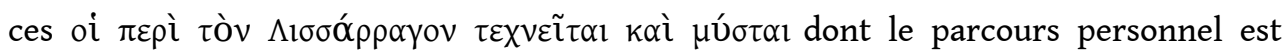
brièvement évoqué dans la liste des auteurs (p. 303-304). 
2 Après une mise en perspective - en guise d'introduction - du parcours de Lissarrague et de sa place dans l'histoire de l'iconographie (V.Azoulay et F. Gherchanoc), cinq parties structurent l'ouvrage. La première ("De l'objet à son dessin au XIX ${ }^{e}$ siècle ») est dédiée aux prémices de l'enseignement de l'iconographie et à Eduard Gerhard, dont tour à tour É. Lehoux et M.-A. Bernard soulignent l'importance du dessin, seule forme de " reproduction » disponible à cette époque et son usage systématisé par cette figure visionnaire du XIX ${ }^{e} s$.

3 La deuxième partie regroupe quatre textes sous l'étiquette des «Rituels au filtre de l'image ». En analysant l'iconographie de quelques vases de mariage, G. Deschodt questionne la connectivité des images entre elles sur le support. Tournant le dos à une lecture strictement sémiotique, elle cherche à donner du sens aux juxtapositions de scènes sur une même image ou entre les images du vase, du col au pied, et entre les faces. Cette recherche de lien sémantique, à travers les liens graphiques, présents ou absents, va parfois un peu loin et prend le risque de créer artificiellement du sens au travers du regard moderne.

4 B. Perriello prend en main la figure de Parthénopée, parangon de la jeunesse belle et flamboyante alliée à la cruauté la plus extrême et cherche à en faire, sans recours aux images mais au travers de l'analyse de textes $d u v^{e} s$., l'emblème même des éphèbes, dont il retrace l'origine et le développement du terme et du concept au IV ${ }^{e}$ s. Cette mise en perspective du caractère clivant de l'adolescence, susceptible de déstructurer l'ordre social permet à l'A. d'arriver aux gymnases, garants d'un cadre canalisateur, dont les cultes garantiraient l'équilibre délicat entre les deux extrêmes de cette jeunesse bouillonnante.

5 Partant de l'utilisation d'un schéma représentatif identique utilisé pour la purification d'Oreste par Apollon, sur la céramique italiote, et pour la guérison de la folie des Proïtides par Mélampous, que l'on peut suivre d'un cratère sicilien du milieu du IV $\mathrm{s}$. à une pâte de verre de Xanten $\left(\mathrm{II}^{\mathrm{e}}-\mathrm{I}^{\mathrm{er}} \mathrm{s}\right.$.) et un camée parisien du $\mathrm{I}^{\mathrm{er}} \mathrm{s}$., Fr. Marzari dresse un catalogue des liens qui rapprochent sémantiquement ces figures a priori sans lien direct, ni dans leur parcours, ni dans les actes qui les mènent à une purification : elle se tourne vers l'âge, l'éloignement de l'oikos, la perte d'identité initiatique, la faute. Cette quête de sens lui permet de souligner la notion de souillure attachée au matricide comme à la folie, mais souillure qu'elle voit non directement comme une impureté religieuse mais comme une souillure politique qui touche parallèlement Oreste comme les filles de Proïtos, celles-ci dans leur refus du mariage lié aux « délires de la puberté ». Ce contexte de sens reconstruit (valable peut-être pour le $\mathrm{IV}^{\mathrm{e}} \mathrm{s}$.) va bien au-delà des images et éloigne quelque peu d'une recherche iconographique. L'analyse de la présence ou absence du porcelet dans ces purifications aurait sans doute permis de mieux ancrer l'analyse dans le concret des choix représentatifs et partant, dans le sens du rituel convoqué.

6 C'est la supplication d'Alcmène, menacée par son époux Amphitryon trompé par Zeus qui forme le cadre d'analyse proposé par M. Pedrina sur la base de vases paestans et italiotes. On soulignera la fine analyse des choix des peintres, qui met en avant tantôt l'autel-refuge tantôt le bûcher-menace qui deviennent autel-bûcher parfois, pour synthétiser l'ambivalence de la situation et du cadre sacré lui-même, les gestes de supplication d'Alcmène qui indiquent la position de l'humain par rapport au divin, qu'il ne voit ni ne "contrôle", la protection de Zeus qui s'exprime graphiquement par un halo ou arc-en-ciel, l'ambivalence des puissances divines représentées, en acteurs 
directs du salut de la jeune femme (Zeus et/ou Éros) ou en signes narratifs de l'origine du drame (Éros et Aphrodite). L'exégèse de la subtilité des procédés iconographiques est aussi le fruit d'une lecture en filigrane des textes présentant l'épisode ; mais l'A. sait éviter le piège de l'asservissement texte-image. On se demandera simplement si le déchiffrement de la présence d'Éros sur différents vases, tantôt vu comme puissance qui sauve (Zeus étant épris d'Alcmène) tantôt comme signe narratif renvoyant à l'origine de l'action peut se justifier au sein de l'image elle-même, sans recours à des textes dont nous ne savons pas dans quelle mesure ils étaient connus des peintres, comme le Peintre de Darius (dont la production, soit dit en passant, est datée par une malencontreuse coquille de 420 et non de 320 ).

7 La troisième partie regroupe des « Personnages mythologiques au figuré » au travers de quatre cas de figure. L'Apollon Hyperpontios d'une célèbre hydrie actuellement au Vatican, voguant au-dessus des eaux sur un trépied ailé, forme le point de départ d'I. Sforza. Cette poursuite analytique et symbolique de l'objet, en l'occurrence le trépied ailé, dont elle souligne en conclusion la fonction polyvalente, la mène à des parallèles dont on a peine à saisir l'opportunité, comme la coupe du soleil emmenant Héraclès ou le char ailé de Triptolème. Une meilleure prise en compte de la syntaxe des objets représentés aurait peut-être permis de mieux mettre en contexte ces « machines volantes » chères à Beazley.

$\mathrm{Ph}$. Arnaud décortique la mise en scène dynamique d'une amphore chalcidienne de Vulci dont nous ne possédons plus que le dessin de 1829. Après avoir attribué le vase au "Peintre des Inscriptions », il fait ressortir de cette mêlée de beau monde grec comme troyen, Trois héros grecs entre la vie et la mort, Ajax triomphant au combat, Diomède se faisant soigner le doigt par Sthénélos et Achille mort. L'absence d'Ulysse et la présence d'Athéna statique et parfaitement immobile au milieu du chaos, dont seuls les serpents de l'égide manifestent un mouvement, menaçant, permettent à l'A. de mettre en perspective la temporalité et la spatialité avec lesquelles joue le peintre. Le sens de la scène se lit d'après Arnaud dans l'anticipation de la colère, de la folie et du suicide d'Ajax induit par la présence d'Athéna. La mort ignominieuse du guerrier, triomphant ici, qui se situe au-delà de l'épisode figuré s'explique par son hybris et son impiété, en deçà de la scène représentée et dont Athéna est comme le rappel. Dès lors, les libertés prises par le peintre avec la tradition (comme une flèche dans le dos d'Achille, et non seulement dans le talon vulnérable de l'invulnérable) s'expliquent par le sens général de la scène: montrer les vicissitudes du guerrier, et finalement de l'homme: du triomphe (Ajax), à la blessure (Diomède) et à la mort (Achille).

En se penchant sur les figures féminines ailées de la figure noire attique, A. Caillaud souligne « l'entre-deux » qu'elles représentent. Ou plutôt les « entre-deux » : placées en figures "quasiment héraldiques» (183) entre deux objets ou deux figures (chars, cavaliers, guerriers, hommes...), elles sont aussi l'expression de la présence divine dans la sphère humaine. Réduisant toute influence d'Hésiode sur la représentation et le mode d'action de ces figures, l'A. souligne combien l'identification de ces êtres ailés comme Nikè, Éris ou Iris importe peu. Il s'agit bien plutôt de reconnaître l'ambiguïté entretenue volontairement par ces schémas, rendant plus important que tout leur rôle d'« entre-deux » multiple, étant elles-mêmes «divines et garantes des frontières qu'elles franchissent » (183).

10 Sur les pinakes de Locres, un schéma qui semble mettre en scène un "Intrus » retient l'attention d'H. Collard. Le satyre en position ambiguë avec un cervidé représenté sur 
un autel dans le schéma du type $3 / 6$ des pinakes locriens semble en effet hors sujet dans ces offrandes qui font une part de choix au mariage et aux rites nuptiaux. En nuançant la fiabilité des dessins, qui sont déjà une interprétation, et sur lesquels la recherche moderne s'appuie, H.Collard retourne aux fragments de reliefs pour relativiser l'interprétation d'accouplement entre le satyre et le cervidé, impossible physiquement du fait de la position jambes tendues du satyre. Partant du constat que l'image portée sur l'autel ne peut être le reflet d'une image « réelle » sur ce support, dont l'archéologie n'a aucune trace avant le $\mathrm{IV}^{\mathrm{e}} \mathrm{s}$, l'A. cherche le sens que le (les) coroplaste(s) a (ont) voulu induire en plaçant dans l'image ce schéma particulier sur ce support précis. Mettant en série, en toute bonne méthode, les images de satyres et de cervidés, elle constate la proximité des poursuites d'animaux et des poursuites amoureuses dont le satyre, par sa mixité, induit le glissement de l'une à l'autre. La maîtrise de l'animal serait une métaphore du mariage, faisant écho aux scènes de rapt que connaissent par ailleurs les pinakes. Il s'agirait, au travers de ce schéma satyre-biche, de faire allusion métaphoriquement à la domestication de la jeune fille, au-delà de la simple référence à l'acte sexuel. L'image insérée dans l'image et sur l'objet rituel le plus central serait ainsi un message direct au couple représenté en train de sacrifier et aux visiteurs du sanctuaire.

11 Les quatrième et cinquième parties («Images en contexte » et « Images fragmentées ») se focalisent davantage sur la construction de l'image ; mais le lecteur intéressé par la religion aurait tort de les laisser de côté : on y rencontre Éros, des héros, tout comme l'autel, et de toute manière, comprendre la religion dans et par les images sous-entend qu'on en maîtrise les processus compositionnels et référentiels.

12 En s'intéressant aux objets qui tombent dans les poursuites amoureuses, la très regrettée Nina Strawczynski démontre que ces objets caractérisent les figures représentées et ne sont pas seulement une fixation graphique d'un instant ponctuel, comme le voulait D. Wannagat. Les fleurs, hydries, couronnes que « lâchent » les jeunes filles poursuivies renvoient à leur identité de jeunes filles justement, que ce soient des figures anonymes ou des héroïnes, comme Circé dépossédée de ses pouvoirs par Ulysse ou Hélène poursuivie par Ménélas. Ce dernier, qui lâche son épée, est caractérisé comme guerrier et homme mûr, tout comme homme reconnaissant un thauma, celui de la force d'Éros qui lui fait perdre ses moyens.

13 Ce sont encore les objets qui sont au centre des réflexions de M. Baggio, le coffret, le collier et le métier à tisser, comme marqueurs là aussi de l'identité des figures (genre, don, séduction). Un va-et-vient entre la première production des vases italiotes (Peintre de Pisticci) et la production attique contemporaine ou légèrement antérieure, autour des scènes de séduction permet à l'auteur d'affirmer que les premiers peintres italiotes «se montrent capable(s) d'interpréter de manière nouvelle et de façon autonome le patrimoine iconographique attique, sans se borner à reproduire des figurations déjà consolidées » (234). Cette indépendance naissante se lirait également dans la possible reprise du schéma de la séduction d'Éryphile pour rappeler ou y superposer différents récits mythiques qui prédominaient dans l'imaginaire antique " (235), comme celui de Pénélope par exemple.

14 C'est à La Mêtis du décor que s'attaque N. Kéi tissant un parallèle entre la polymorphie et la polyvalence du décor végétal (palmettes) qui envahit les vases pour entrer même à l'intérieur des scènes figurées parfois comme agent narratif et les qualités prêtées au poulpe. Cette polymorphie qui devient Mêtis par glissement analogique du polymorphon 
au polytropon dont Ulysse est le champion mène l'auteur, un peu hardiment, à faire des motifs dits ornementaux des vecteurs de valeurs liées à l'intelligence.

Revenant sur le lièvre comme cadeau homo-érotique bien connu, E. Reshetnikova reprend les représentations d'érastes et d'éromènes sur la céramique attique des $\mathrm{VI}^{\mathrm{e}}$ et $\mathrm{V}^{\mathrm{e}}$ siècles en soulignant les marqueurs usuels des identités et rôles dévolus à chacun. Son attention se focalise en particulier sur les scènes qui ne présentent qu'un seul des deux acteurs du couple, avec un lièvre. En passant par les représentations dans lesquelles les marqueurs habituels semblent brouillés, et en soulignant l'existence d'érastes imberbes, d'éromènes barbus, avec canne, etc., l'A. insiste sur l'ambiguïté voulue entre les deux statuts, et sur le lièvre comme cadeau pouvant aller dans les deux sens. Une mise en perspective chronologique aurait permis de mieux saisir le fondement et la pertinence de cette analyse.

La cinquième partie regroupe, enfin, deux analyses de l'image dans sa construction, ses références et dans l'usage que nous en faisons au travers du concept d'image « incomplète ». V. Zachari s'interroge sur les scènes à l'autel sans figure humaine pour "étudier les manières dont l'espace figuratif se construit et s'organise sans embrayeur " (258). Il peut sembler contre-productif de placer d'entrée de jeu, et a contrario, la présence humaine dans l'image comme un moteur (« embrayeur»), et donc conséquemment son absence comme un problématique espace vide. Mais l'analyse se concentre très vite sur les traces de l'activité de l'homme (pinakes, bandelettes, feu ou traces de sang sur l'autel), pour arriver à la conclusion que la puissance de la gestualité est mise en valeur par ces signes et que l'espace, même sans figure humaine, en porte la présence en filigrane. Mais, en fait, le sanctuaire en lui-même, en dehors de toute action ou présence évoquées, dans sa simple existence comme lieu construit et organisé pour la rencontre avec le divin, n'est-il pas lui-même la plus grande trace de l'existence, de la présence et du statut de l'homme?

N. Dietrich, comme une cerise sur le gâteau - ou das Tüpfelchen auf dem $i-$, nous emporte dans un brillant essai sur la "valeur heuristique du fragment». Pris comme métaphore de notre situation face à la documentation antique, le concept de fragment permet tout d'abord à l'A. de pointer les biais de perspectives que nous endossons à cause de nos «lacunes" en matière documentaire que nous tentons de compenser par une concentration abusive sur ce qui reste. Et par là même il met en lumière des questions fondamentales de méthode, en investissant jusqu'au bout ce qui devient dans son analyse, un puissant outil critique. Inversant les perspectives traditionnelles, il fait $\mathrm{du}$ fragment non le signe d'une incomplétude, mais une chance heuristique au potentiel insoupçonné. Entre focalisation forcée et féconde, reconnaissance du hasard de la documentation, dialogue de l'essentiel et du marginal - et de soi à soi -, le fragment ainsi conçu (re)dessine une méthodologie appliquée. Il n'est pas possible de redonner ici toute la palette des fines remarques et analyses qui construisent le propos et interrogent sans cesse notre façon d'appréhender le matériel antique Nous retiendrons dans cette brève relation, forcément fragmentaire, combien la puissance d'une réflexion herméneutique, portée sur un objet matériel et iconographique, peut apporter au chercheur actuel, quel qu'en soit le domaine, dans la prise en compte de sa position, et en fin de compte dans son identité scientifique.

Finir ce bouquet d'hommages sur une telle réflexion fondamentale et voir se dessiner, en conclusion avec É. Lehoux et $\mathrm{V}$. Zachari, les axes de force révélés par le tissage des dix-neuf textes ${ }^{1}$ rassemblés ne peut que souligner la puissance d'une carrière et d'un 
enseignement, dans sa générosité et sa quête inlassable et fécondante. Les bénéfices de l'iconographie ne se limitent pas aux images.

\section{NOTES}

1. La table des matières est reprise ci-dessous, p. 361.

\section{AUTEURS}

\section{ANNE-FRANÇOISE JACCOTTET}

Université de Genève 